\title{
Asymptomatic infection in individuals from the municipality of Barcelos (Brazilian Amazon) is not associated with the anti-Plasmodium falciparum glycosylphosphatidylinositol antibody response
}

\author{
Larissa Rodrigues Gomes ${ }^{1,2}$, Paulo Renato Rivas Totino ${ }^{1,2}$, Maria Carmen Arroyo Sanchez ${ }^{3}$, \\ Elsa Paula da Silva Kaingona Daniel ${ }^{1,2,4}$, Cristiana Santos de Macedo ${ }^{5}$, Filomeno Fortes ${ }^{6}$, \\ José Rodrigues Coura ${ }^{7}$, Silvia Maria Di Santi ${ }^{8}$, Guilherme Loureiro Werneck ${ }^{9}$, \\ Martha Cecilia Suárez-Mutis ${ }^{7}$, Maria de Fátima Ferreira-da-Cruz ${ }^{1,2}$, Cláudio Tadeu Daniel-Ribeiro',2/+ \\ ${ }^{1}$ Laboratório de Pesquisa em Malária ${ }^{5}$ Laboratório de Microbiologia Celular ${ }^{7}$ Laboratório de Doenças Parasitárias, Instituto Oswaldo Cruz \\ ${ }^{2}$ Centro de Pesquisa, Diagnóstico e Treinamento em Malária-Fiocruz, Rio de Janeiro, RJ, Brasil \\ ${ }^{3}$ Laboratório de Soroepidemiologia e Imunobiologia, Instituto de Medicina Tropical de São Paulo, São Paulo, SP, Brasil \\ ${ }^{4}$ Hospital Central Dr Antônio Agostinho Neto, Lubango, Angola ${ }^{6}$ Instituto Nacional de Saúde Pública, Luanda, Angola \\ ${ }^{8}$ Centro de Pesquisas em Malária, Superintendência de Controle de Endemias, São Paulo, SP, Brasil \\ ${ }^{9}$ Núcleo de Estudos em Malária, Instituto de Medicina Social, Universidade do Estado do Rio de Janeiro, Rio de Janeiro, RJ, Brasil
}

Anti-glycosylphosphatidylinositol (GPI) antibodies (Abs) may reflect and mediate, at least partially, anti-disease immunity in malaria by neutralising the toxic effect of parasitic GPI. Thus, we assessed the anti-GPI Ab response in asymptomatic individuals living in an area of the Brazilian Amazon that has a high level of malaria transmission. For comparative purposes, we also investigated the Ab response to a crude extract prepared from Plasmodium falciparum, the merozoite surface protein (MSP) 3 antigen of $\mathrm{P}$. falciparum and the MSP 1 antigen of Plasmodium vivax (PvMSP1- $\left.{ }_{10}\right)$ in these individuals and in Angolan patients with acute malaria. Our data suggest that the Ab response against $\mathrm{P}$. falciparum GPI is not associated with P. falciparum asymptomatic infection in individuals who have been chronically exposed to malaria in the Brazilian Amazon. However, this Ab response could be related to ongoing parasitaemia (as was previously shown) in the Angolan patients. In addition, our data show that PvMSP1- ${ }_{19}$ may be a good marker antigen to reflect previous exposure to Plasmodium in areas that have a high transmission rate of $\mathrm{P}$. vivax.

Key words: Plasmodium falciparum - Plasmodium vivax - glycosylphosphatidylinositol -

asymptomatic infection - Brazilian Amazon - Angola

Glycosylphosphatidylinositol (GPI), a glycolipid that is ubiquitously present in eukaryotic cells, has been implicated in the pathogenesis of many parasitic diseases (Debierre-Grockiego 2010). In malaria, the GPI structures of Plasmodium falciparum are conserved among isolates (Berhe et al. 1999). This molecule is released at the end of the schizogonic process and induces an important host immune response, which includes the production of pro-inflammatory cytokines, such as tumour necrosis factor- $\alpha$, interleukin (IL)-1 and IL-6 and the augmented expression of cell adhesion molecules on

doi: 10.1590/0074-0276108062013018

Financial support: POM-IOC, FIOCRUZ, FAPERJ [fellowships to CTDR (E-26/102.420/2009) and MFFC (E-26/102.419/2009) as Cientistas do Nosso Estado]

CTDR, GLW, JRC and MFFC are recipients of a Research Productivity Fellowship from CNPq, LRG received a Master of Sciences fellowship from CAPES.

+Corresponding author: ctdr@uol.com.br

Received 14 August 2012

Accepted 16 May 2013 macrophages and vascular endothelial cells (Schofield et al. 1996). The clinical presentation of the toxaemic syndrome that accompanies the septicaemia caused by Gram-negative bacteria (van Amersfoort et al. 2003) and the usual triad of clinical symptoms of malaria (fever, chills and sweating, which are commonly accompanied by headache) are similar and can result from the proinflammatory responses that are induced by lipopolysaccharide and P. falciparum GPI, respectively. These pro-inflammatory responses can also account for the development of complications, such as severe anaemia and the cerebral form of malaria. Indeed, the administration of P. falciparum GPI in mice induces malaria-like symptoms (Schofield et al. 1993) and immunisation with a non-toxic moiety of GPI that elicits an anti-GPI antibody (Ab) response can prevent malaria pathology and death caused by experimental Plasmodium berghei ANKA [because of the discovery of this murine African Plasmodium in Katanga (NK for New York-Katanga) and in Kasapa (ANKA for Antwerpen-Kasapa (Vincke \& Bafort 1968)] infection in mice (Schofield et al. 2002).

Studies performed in areas of high malaria transmission in Africa and Indonesia have demonstrated the potential protective role of the anti-GPI response in malaria (Naik et al. 2000) and the association of the anti-GPI re- 
sponse with age (Naik et al. 2000, Keenihan et al. 2003). According to these studies, anti-GPI Ab would reflect and mediate, at least partially, anti-disease immunity in malaria by neutralising the toxic effect of parasitic GPI (de Souza et al. 2010). However, studies by Boutlis et al. (2002) and Cissoko et al. (2006) found no relationship between anti-GPI Ab levels and clinical protection from the disease. Here, we investigate the anti-GPI Ab response in asymptomatic individuals living in Barcelos, an area of the Brazilian Amazon with high endemic levels of malaria. Twenty-eight asymptomatic individuals ranging from three-50 years of age $($ mean $=22)$ with either $P$. falciparum $(\mathrm{n}=13)$ or Plasmodium vivax $(\mathrm{n}=$ 15) infections and 12 non-infected individuals ranging from three-66 years of age $($ mean $=16)$ with no declared previous history of malaria were recruited in Barcelos, which has an annual parasitic index (API) of 70.9 cases of malaria per 1,000 inhabitants. In a fieldwork conducted from 2004-2007 (Suárez-Mutis 2007), these asymptomatic individuals tested positive for the presence of $P$. falciparum and $P$. vivax DNA by polymerase chain reaction (PCR) (Snounou 1996). None of these asymptomatic individuals were positive when tested by microscopic examination at the time of blood collection or presented any symptoms during the 30 days preceding or the 30 days succeeding the PCR diagnosis; none of these individuals received anti-malarial treatment during this timeframe. All individuals reported previous malaria attacks $(3-12$; mean $=7.3)$ from both $P$. falciparum and $P$. vivax. Comparatively, we also studied 97 individuals ranging from $15-67$ years of age (mean $=26)$ with noncomplicated $P$. falciparum malaria that was diagnosed by microscopic examination (parasitaemia ranging from $500-3,000$; mean $=1,320$ parasites $/ \mu \mathrm{L}$ ). These individuals lived in Lubango, which is an area of intense malaria transmission in Angola (API = higher than 100 cases per 1,000 inhabitants, depending on the season) and were diagnosed and treated at the Central Lubango Hospital. Brazilian individuals living in the non-endemic southeast region that had never visited malaria-endemic areas were included in the study to establish a threshold (or cut-off) value for ELISA detection. The statistical analysis was performed using Epi Info ${ }^{\circledR}$ software v. 7 (Centers for Disease Control and Prevention). This study was approved by Brazilian (Fiocruz, state of Rio de Janeiro, CEP157/02) and Angolan Ethical Committees (National Malaria Control Program).

The presence of anti-GPI Ab in plasma samples was detected by ELISA (Naik et al. 2000) using P. falciparum GPI extracted from in vitro culture; the extraction of GPI was done according to the method described by Berhe et al. (1999) that maintains the GPI structure required for the recognition of the native molecule. Briefly, because late trophozoites (30-40 h) have been shown to have the largest GPI pool (Schmidt et al. 1998), GPI was extracted from $1 \times 10^{9}$ trophozoites in a onestep process that allowed the simultaneous extraction of all GPI intermediates and free GPI anchors. A mixture of chloroform:methanol $(1: 1, \mathrm{v}: \mathrm{v})$ was added to the sediment so that the final proportion of solvents (which comprised the aqueous phase of the crude parasite GPI extract) was 10:10:3 (v:v:v) chloroform:methanol:water (C:M:W). The suspension was sonicated for $5 \mathrm{~min}$ and then centrifuged $\left(18,000 \mathrm{~g}\right.$ at $25^{\circ} \mathrm{C}$ for $\left.5 \mathrm{~min}\right)$. The supernatant was collected and the sediment was subsequently extracted two more times with $1 \mathrm{~mL}$ C:M:W (10:10:3, $\mathrm{v}: \mathrm{v}: \mathrm{v})$. All of the supernatants were pooled and dried in a SpeedVac vacuum evaporator to obtain C:M:W erythrocytic extracts. To remove water-soluble contaminants, such as nucleotides and phosphate sugars and other lipids, such as phospho and sphingolipids, "Folch washing" was performed (Folch et al. 1957, Field \& Menon 1992). The lipid extract was then resuspended in 1:1 (v:v) C:M and $1 / 5$ volume of $4 \mathrm{mM} \mathrm{MgCl} 2$ was added. The suspension was mixed and centrifuged $\left(18,000 \mathrm{~g}\right.$ at $25^{\circ} \mathrm{C}$ for $5 \mathrm{~min})$. The upper phase was removed and the organic phase was washed twice with $1 / 2$ volume of an artificial upper phase (C:M:W:1 $\mathrm{M} \mathrm{MgCl}_{2}, 6: 96: 94: 0.336$, v:v:v:v). The C:M extracts were vacuum-dried and submitted to a butanol:water phase partition. The dried C:M extracts were resuspended in $200 \mu \mathrm{L}$ water-saturated n-butanol and then the same volume of water was added. After mixing, the extracts were centrifuged $\left(18,000 \mathrm{~g}\right.$ at $25^{\circ} \mathrm{C}$ for $5 \mathrm{~min}$ ) and the aqueous phase was removed. This process was repeated twice. The aqueous phases were then pooled and back-washed with $200 \mu \mathrm{L}$ water-saturated n-butanol. Finally, the butanol phases were pooled, yielding the entire $P$. falciparum GPI content (Gerold et al. 1994), which was used as an antigen in this study.

Ideally, after the collection of the butanol phases, the GPI extraction is followed by an additional purification step using metabolic $\left[{ }^{3} \mathrm{H}\right]$ glucosamine to label the GPI for high-performance liquid chromatography. However, $\left[{ }^{3} \mathrm{H}\right]$ glucosamine was not available at our institution due to environmental restrictions. $\left[{ }^{3} \mathrm{H}\right]$ glucosamine has a halflife of approximately 13 years and is potentially harmful to health and the environment. Because GPIs are, so far, the only known glycoconjugates present in P. falciparum parasites (Gerold et al. 1994, Schmidt et al. 1998, Berhe et al. 1999, Azzouz et al. 2008) and because the solvent specificity for GPI extraction (C:M:W) together with the subsequent washing step assured the elimination of eventual contaminants (Folch et al. 1957, Field \& Menon 1992), we decided to use the $P$. falciparum GPI pool without an additional radioactivity purification step. To circumvent false-positive ELISA results, additional "GPI controls", obtained from non-parasitised erythrocytes (treated or not with saponin and submitted to the same extraction protocol), were utilised; only one saponin-treated and two non-saponin-treated non-parasitised erythrocyte samples out of 137 were found to be positive.

There are no reports of $P$. vivax GPI extraction and asymptomatic $P$. vivax-infected individuals were also tested for $P$. falciparum GPI because they had previous $P$. falciparum infections. ELISA positivity was calculated using the index of reactivity (IR) ratio (the absorbance value of each sample divided by the cut-off value; the samples with a ratio of $\geq 1$ were considered positive). The cut-off value for each reaction was calculated as the mean plus two times the standard deviation of a sera panel from 20 clinically healthy individuals who neither lived in nor visited malaria-endemic areas. 
The frequency and reactivity of anti-GPI $\mathrm{Ab}$ in individuals from Barcelos with asymptomatic P. falciparum infection were not significantly different $(\mathrm{p}>0.05)$ from those recorded in non-infected individuals from the same area without a previous history of malaria (Figure, Table). The same was true for $P$. vivax asymptomatic carriers. Because GPI levels decline with time after infection, we divided the individuals from Barcelos into three groups according to the time elapsed after their last malaria attack caused by $P$. falciparum infection $(\leq 2$ months, $>2$ and $\leq 6$ months and $>6$ and $\leq 12$ months) (n $=28$ ). The anti-GPI Ab levels in these three groups were then analysed. Anti-GPI Ab levels ranged from 1.011.42 IR (mean =1.2) and were independent of the time of infection. Therefore, this parameter does not seem to be relevant in determining the anti-GPI Ab level. Conversely, patients from Lubango with P. falciparum malaria had a significantly higher anti-GPI Ab frequency $(\mathrm{p}<0.0001$, analysis of variance) and reactivity $(\mathrm{p}<$ 0.0001 , Student's $t$ test) (Figure, Table). These contrasting data do not support the hypothesis that the anti-GPI $\mathrm{Ab}$ level reflects clinical immunity to malaria (Naik et al. 2000, de Souza et al. 2002, Keenihan et al. 2003), but are in accordance with the conclusions of Boutlis et al. (2002) and Cissoko et al. (2006). Although the anti-GPI response was not higher in asymptomatic infection, this response seemed to be related to age; positive individuals were older $(33.8 \pm 7.2)$ than negative individuals (18.6 $\pm 14.5)(\mathrm{p}<0.03$, Student's $t$ test), indicating a possible association with repeated and chronic infection, which accompany aging in endemic areas. Indeed, the anti-GPI response tended to correlate with the number of past episodes of malaria, even though this correlation did not reach statistical significance; positive individuals had more previous malaria attacks $(8.8 \pm 2.7)$ than the negative individuals $(6.0 \pm 3.2)(\mathrm{p}<0.08$, Student's $t$ test). Moreover, it has already been reported that the prevalence and concentration of anti-GPI Ab in hyperendemic areas of malaria increase with age (Naik et al. 2000, Boutlis et al. 2002, de Souza et al. 2002). Conversely, in the symptomatic malaria patients from Lubango, the GPI immune response was independent of age, but was positively correlated with parasitaemia $(r=0.398 ; p=0.010$, Spearman's rank correlation), which could have resulted from a booster effect of parasitic antigens during acute infection. Similar observations have been described by de Souza et al. (2002) and Cissoko et al. (2006), who reported that in cases of clinical malaria, anti-GPI $\mathrm{Ab}$ can be augmented in a parasitaemia-dependent manner. Considering our results here, we hypothesise that in the patients with uncomplicated malaria from Lubango, GPI Abs could protect against the development of severe malaria because circulating anti-GPI Abs can neutralise the toxic effects of parasitic GPI (Naik et al. 2000).

To further characterise the previous exposure of the studied individuals to Plasmodium, we also evaluated the $\mathrm{Ab}$ response to a crude $P$. falciparum extract by ELISA [obtained according to Sanchez et al. (1993)] as well as to the recombinant merozoite surface proteins (MSP) of $P$. falciparum ( $P f \mathrm{MSP} 3$ ) (a kind gift from Pierre Druilhe from Vac-4-All) and $P$. vivax ( $P v \mathrm{MSP} 1_{19}$ ) (produced by Arianni Rondelli Sanchez and Eduardo Milton RamosSanchez at the Institute of Tropical Medicine, state of São Paulo, Brazil), as described elsewhere (Theisen et al. 2001, Cunha et al. 2001, respectively). The Ab levels to all antigens in the asymptomatic individuals were

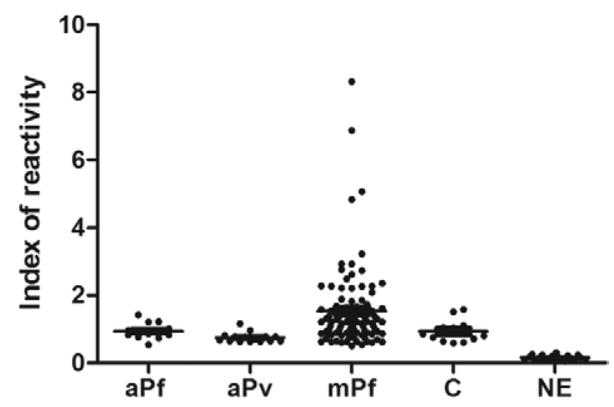

Antibody $(\mathrm{Ab})$ reactivity against Plasmodium falciparum glycosylphosphatidylinositol (GPI) in serum samples from asymptomatic $P$. falciparum (aPf) and Plasmodium vivax (aPv) infected and control (C) individuals from Barcelos (Brazilian Amazon), as well as from malarious P. falciparum (mPf) patients from Lubango (Angola). Samples were diluted at 1:100 and the Ab reactivity to $P$. falciparum GPI (IgG) was detected by ELISA. Individuals never exposed (NE) to malaria were used as negative controls.

TABLE

Frequency of antibodies (Abs) detected by ELISA against glycosylphosphatidylinositol (GPI), the crude extract (PfAg) and the recombinant protein merozoite surface proteins (MSP) of Plasmodium falciparum (PfMSP3) and the recombinant protein of Plasmodium vivax $\left(P v \mathrm{MSP} 1{ }_{19}\right)$

Frequency of Abs

$(\%)$

\begin{tabular}{lcccc}
\cline { 2 - 4 } Groups & GPI & PfAg & PfMSP3 & PvMSP1- ${ }_{19}$ \\
\hline Asymptomatics (Barcelos) & 18 & 96 & 46 & 86 \\
Non-infected controls (Barcelos) & 42 & 50 & 42 & 17 \\
Malaria patients (Lubango) & 65 & 70 & 44 & 2 \\
\hline
\end{tabular}


significantly related to age (crude antigen: $\mathrm{r}=0.523, \mathrm{p}=$ 0.004, $P f \mathrm{MSP} 3: \mathrm{r}=0.386, \mathrm{p}=0.04$ and $P v \mathrm{MSP}_{-}{ }_{19}: \mathrm{r}=$ $0.496, \mathrm{p}=0.007$, Spearman's rank correlation), indicating that, in a scenario of supposed premunition to malaria in areas of intense disease transmission (including those in the Brazilian Amazon), antiparasitic responses can be associated with age and the consequent cumulative exposure to infection. This idea has been classically reported for the hyperendemic areas of Africa (Soe et al. 2001, Dodoo et al. 2008). In Brazilian endemic areas, it has also been shown that the $\mathrm{Ab}$ response to $P v \mathrm{MSP} 1_{-19}$ increases with age and/or time of exposure to malaria (Braga et al. 2002, Nogueira et al. 2006). Additionally, the $\mathrm{Ab}$ response has even been linked to the absence of clinical symptoms (Nogueira et al. 2006). However, in the African patients with acute $P$. falciparum malaria, the Abs against the $P$. falciparum extract and PfMSP3 were associated with parasitaemia, supporting our data obtained for GPI that suggest a rather non-protective immune response linked to acute infection.

An interesting issue raised in our study was the observation of a high prevalence of $\mathrm{Ab}$ to $P$. falciparum blood-stage antigens, including $P f \mathrm{MSP} 3$, among the control individuals from Barcelos (Table). This was the first time it was studied in a Brazilian endemic area. These individuals presenting $\mathrm{Ab}$ to MSP3, but not $\mathrm{Ab}$ to GPI never reported previous malaria episodes nor were found to be infected with plasmodia by PCR analysis. The similar positivity of anti- $P f \mathrm{MSP} 3 \mathrm{Ab}$ in both Brazilians who had been exposed to infection, but did not have a previous history of malaria infection and Angolans who presented with malaria with positive thick blood smears is quite surprising and could be explained in a number of ways: (i) the Brazilian individuals could have had atypical malaria attacks that were not recognised as classical manifestations of the disease, (ii) PfMSP3 could have cross-reacted with molecules from other microorganisms that are present in the region or (iii) the anti-Pf MSP $3 \mathrm{Ab}$ response might not have accurately reflected earlier exposure of the African individuals to the parasite.

In this study, the anti- $P v \mathrm{MSP} 1-{ }_{19} \mathrm{Ab}$ response seems to adequately reflect previous experience with $P$. vivax. These observations are in agreement with a study conducted in another malarious Brazilian region (Cotijuba, Belém, state of Pará) with exclusive transmission of $P$. vivax, in which $50 \%$ of individuals with no past history of the disease presented Abs against the blood stages of $P$. vivax, as measured by indirect fluorescent antibody test, while only approximately $6 \%$ of these individuals presented Abs against $P v \mathrm{MSP}_{-}{ }_{19}$, as measured by ELISA (Soares et al. 1999). In addition to the low prevalence of anti- $P v \mathrm{MSP} 1-{ }_{19} \mathrm{Ab}(17 \%)$ recorded in the control subjects from Barcelos, we observed a very low prevalence (2\%) of these Abs among African patients living in areas where P. falciparum is highly prevalent. In contrast, these Abs were present in $86 \%$ of the asymptomatic infected subjects from Barcelos, where P. vivax is the major malariacausing species. These data suggest that the assessment of positivity to Abs reacting with $P v \mathrm{MSP} 1-_{19}$ could be valuable to define exposure to $P$. vivax in endemic areas.
In summary, the data presented here, which for the first time address the anti- $P$. falciparum GPI immune response in Brazil, indicate that the $\mathrm{Ab}$ response against $P$. falciparum GPI is not associated with asymptomatic infection in individuals who have been chronically exposed to malaria in the Brazilian Amazon. These data are in agreement with data from other African endemic areas (de Souza et al. 2002, Cissoko et al. 2006). Supplementary studies with a higher number of asymptomatic individuals comparatively to patients with acute malaria from the same origin will increase the understanding of the relationship between anti-GPI Ab responses and asymptomatic infection.

\section{REFERENCES}

Azzouz N, Gerold P, Schwarz RT 2008. Metabolic labeling and structural analysis of glycosylphosphatidylinositols from parasitic protozoa. Methods Mol Biol 446: 183-198.

Berhe S, Schofield L, Schwarz RT, Gerold P 1999. Conservation of structure among glycosylphosphatidylinositol toxins from different geographic isolates of Plasmodium falciparum. Mol Biochem Parasitol 103: 273-278.

Boutlis CS, Gowda DC, Naik RS, Maguire GP, Mgone CS, Bockarie MJ, Lagog M, Ibam E, Lorry K, Anstey NM 2002. Antibodies to Plasmodium falciparum glycosylphosphatidylinositols: inverse association with tolerance of parasitemia in Papua New Guinean children and adults. Infect Immun 70: 5052-5057.

Braga EM, Barros RM, Reis TA, Fontes CJ, Morais CG, Martins MS, Krettli AU 2002. Association of the IgG response to Plasmodium falciparum merozoite protein (C-terminal $19 \mathrm{kD}$ ) with clinical immunity to malaria in the Brazilian Amazon Region. Am J Trop Med Hyg 66: 461-466.

Cissoko Y, Daou M, Lyke KE, Dicko A, Diarra I, Kone A, Guindo A, Traore K, Krishnegowda G, Diallo DA, Doumbo OK, Plowe CV, Gowda DC, Sztein MB 2006. Serum antibody levels to glycosylphosphatidylinositols in specimens derived from matched Malian children with severe or uncomplicated Plasmodium falciparum malaria and healthy controls. Am J Trop Med Hyg 75: 199-204.

Cunha MG, Rodrigues MM, Soares IS 2001. Comparison of the immunogenic properties of recombinant proteins representing the Plasmodium vivax vaccine candidate MSP1(19) expressed in distinct bacterial vectors. Vaccine 20: 385-396.

de Souza JB, Runglall M, Corran PH, Okell LC, Kumar S, Gowda DC, Couper KN, Riley EM 2010. Neutralization of malaria glycosylphosphatidylinositol in vitro by serum IgG from malariaexposed individuals. Infect Immun 78: 3920-3929.

de Souza JB, Todd J, Krishegowda G, Gowda DC, Kwiatkowski D, Riley EM 2002. Prevalence and boosting of antibodies to Plasmodium falciparum glycosylphosphatidylinositols and evaluation of their association with protection from mild and severe clinical malaria. Infect Immun 70: 5045-5051.

Debierre-Grockiego F 2010. Glycolipids are potential targets for protozoan parasite diseases. Trends Parasitol 26: 404-411.

Dodoo D, Aikins A, Kusi KA, Lamptey H, Remarque E, Milligan P 2008. Cohort study of the association of antibody levels to AMA1, MSP119, MSP3 and GLURP with protection from clinical malaria in Ghanaian children. Malar J 7: 142-147.

Field MC, Menon AK 1992. Biosynthesis of glycosyl-phosphatidylinositol membrane protein anchors. In NM Hooper, AJ Turner (eds.), Lipid modification of proteins - A practical approach, IRL Press, Oxford, p. 155-190. 
Folch J, Lees M, Sloane Stanley GH 1957. A simple method for the isolation and purification of total lipids from animal tissues. $J$ Biol Chem 226: 497-509.

Gerold P, Dieckmann-Schuppert A, Schwarz RT 1994. Glycosylphosphatidylinositols synthesized by asexual erythrocytic stages of the malarial parasite, Plasmodium falciparum. Candidates for plasmodial glycosylphosphatidylinositol membrane anchor precursors and pathogenicity factors. J Biol Chem 269: 2597-2606.

Keenihan SN, Ratiwayanto S, Soebianto S, Krisin Marwoto H, Krishnegowda G, Gowda DC, Bangs MJ, Fryauff DJ, Richie TL, Kumar S, Baird JK 2003. Age-dependent impairment of IgG responses to glycosylphosphatidylinositol with equal exposure to Plasmodium falciparum among Javanese migrants to Papua, Indonesia. Am J Trop Med Hyg 69: 36-41.

Naik RS, Branch OH, Woods AS, Vijaykumar M, Perkins DJ, Nahlen BL, Lal AA, Cotter RJ, Costello CE, Ockenhouse CF, Davidson EA, Gowda DC 2000. Glycosylphosphatidylinositol anchors of Plasmodium falciparum: molecular characterization and naturally elicited antibody response that may provide immunity to malaria pathogenesis. J Exp Med 192: 1563-1576.

Nogueira PA, Alves FP, Fernandez-Becerra C, Pein O, Santos NR, da Silva LHP, Camargo EP, del Portillo HA 2006. A reduced risk of infection with Plasmodium vivax and clinical protection against malaria are associated with antibodies against the $\mathrm{N}$ terminus, but not the $\mathrm{C}$ terminus of merozoite surface protein 1. Infect Immun 74: 2726-2733.

Sanchez MCA, Avila SLM, Quartier-Oliveira VP, Ferreira AW 1993. Malaria serology: performance of six Plasmodium falciparum antigen extracts and of three ways of determining serum titers in IgG and IgM-ELISA. Rev Inst Med Trop Sao Paulo 35: 495-502.

Schmidt A, Schwarz RT, Gerold P 1998. Plasmodium falciparum: asexual erythrocytic stages synthesize two structurally distinct free and protein-bound glycosylphosphatidylinositols in a maturation-dependent manner. Exp Parasitol 88: 95-102.

Schofield L, Hewitt MC, Evans K, Siomos MA, Seeberger PH 2002. Synthetic GPI as a candidate anti-toxic vaccine in a model of malaria. Nature 418: 785-789.

Schofield L, Novakovic S, Gerold P, Schwarz RT, McConville MJ, Tachado SD 1996. Glycosylphosphatidylinositol toxin of Plas- modium up-regulates intercellular adhesion molecule-1, vascular cell adhesion molecule-1, and E-selectin expression in vascular endothelial cells and increases leukocyte and parasite cytoadherence via tyrosine kinase-dependent signal transduction. $J$ Iтmиnol 156: 1886-1896.

Schofield L, Vivas L, Hackett F, Gerold P, Schwarz RT, Tachado S 1993. Neutralizing monoclonal antibodies to glycosylphosphatidylinositol, the dominant TNF-alpha-inducing toxin of Plasmodium falciparum: prospects for the immunotherapy of severe malaria. Ann Trop Med Parasitol 87: 617-626.

Snounou G 1996. Detection and identification of the four malaria parasite species infecting humans by PCR amplification. Methods Mol Biol 50: 263-291.

Soares IS, Oliveira SG, Souza JM, Rodrigues MM 1999. Antibody response to the $\mathrm{N}$ and $\mathrm{C}$-terminal regions of the Plasmodium vivax merozoite surface protein 1 in individuals living in an area of exclusive transmission of $P$. vivax malaria in the North of Brazil. Acta Trop 72: 13-24.

Soe S, Khin SA, Htay A, Nay W, Tin A, Than S 2001. Premunition against Plasmodium falciparum in a malaria hyperendemic village in Myanmar. Trans $R$ Soc Trop Med Hyg 95: 81-84.

Suárez-Mutis MC 2007. Epidemiologia da malária em comunidades do Rio Padauiri, médio Rio Negro, uma área de extrativismo vegetal da piaçaba no estado do Amazonas, Brasil, $\mathrm{PhD}$ Thesis, Fundação Oswaldo Cruz, Rio de Janeiro, 169 pp.

Theisen M, Dodoo D, Toure-Balde A, Soe S, Corradin G, Koram KK, Kurtzhals JA, Hviid L, Theander T, Akanmori B, Ndiaye M, Druilhe P 2001. Selection of glutamate-rich protein long synthetic peptides for vaccine development: antigenicity and relationship with clinical protection and immunogenicity. Infect Immun 69: 5223-5229.

van Amersfoort ES, van Berkel TJ, Kuiper J 2003. Receptors, mediators and mechanisms involved in bacterial sepsis and septic shock. Clin Microbiol Rev 16: 379-414.

Vincke LH, Bafort F 1968. Results of 2 years of observation of the cyclical transmission of Plasmodium berghei. Ann Soc Belges Med Trop Parasitol Mycol 48: 439-454. 